\title{
Hecke action and the degree of the modular parameterization
}

\author{
by
}

\author{
ArJune Budhram (New York, NY)
}

1. Introduction. Let $\Gamma \subset \mathrm{PSL}_{2}(\mathbb{R})$ be a Fuchsian group of the first kind acting on the complex upper half plane $\mathcal{H}$ and $X$ the resulting modular curve, i.e. the complex points of $X$ can be described analytically as a compactification of the quotient $\Gamma \backslash \mathcal{H}$ by adding cusps of $\Gamma$. Further, suppose we have a non-constant map $\varphi: X \rightarrow E$, where $E$ is some elliptic curve defined over $\mathbb{Q}$, i.e. $E$ is modular. The pull-back of the unique (up to scalar multiplication) holomorphic differential on $E$ is the differential $2 \pi i f(\tau) d \tau$, where $f: \mathcal{H} \rightarrow \mathbb{C}$ is a holomorphic cuspform of weight 2 on $\Gamma$ (we assume that the Manin constant is 1). Recent results ([1], [2], [6]-[8]) show that every elliptic curve $E$ defined over $\mathbb{Q}$ is modular, i.e. there exist $\varphi: X_{0}(N) \rightarrow E$ and $f$ related to $E$ as above, the integer $N$ being the conductor of $E$ and $f$ is a newform of level $N$. We refer to the map $\varphi$ as "the modular parameterization."

In [9], Zagier computes the Petersson norm of the cusp-form $f$, related to $E$ as above, in two different ways. He thus obtains a formula for the degree of the modular parameterization in terms of period integrals on the modular curve. In this paper we extend the ideas presented in [9] to obtain another formula for the degree of the modular parameterization and one for the Hecke eigenvalues (Fourier coefficients) of the the cusp form $f$. The degree of the map $\varphi: X_{0}(N) \rightarrow E$ is of interest for it was shown by Frey [3] that Szpiro's conjecture (relating the conductor and discriminant of elliptic curves over $\mathbb{Q}$ ) is equivalent to the degree of the map $\varphi$ having polynomial growth in the level $N$. The Hurwitz formula gives no information about the degree since the Euler characteristic of $E$ is zero.

Acknowledgments. I wish to thank Dorian Goldfeld for many helpful conversations.

2000 Mathematics Subject Classification: Primary 11G18. 
2. Fundamental domains and the homomorphism $C_{f}$. Let $\Gamma \subset$ $\mathrm{PSL}_{2}(\mathbb{R})$ be a Fuchsian group of the first kind acting on the complex upper half plane $\mathcal{H}$ and $\mathcal{F}=\Gamma \backslash \mathcal{H}^{*}$ be a fundamental domain for the action $\left(\mathcal{H}^{*}=\mathcal{H} \cup\{\right.$ cusps of $\left.\Gamma\}\right)$. We view $\mathcal{F}$ as a hyperbolic polygon with vertices being interior or boundary points of $\mathcal{H}$ and having a finite number of edges which are identified in pairs in $\mathcal{F}$. The vertices $\left\{P_{j}\right\}_{j \in J}$ are labeled in such a way that $P_{j+1}$ is the successor of $P_{j}$ in the natural orientation. Let $e_{j}$ denote the edge $P_{j} P_{j+1}, e_{j^{*}}$ the edge with which it gets identified and $\gamma_{j} \in \Gamma$ the element that identifies them. The map $*: J \rightarrow J$ is an involution on $J$ and the matrices $\gamma_{j}$ are generators of $\Gamma$ satisfying $\gamma_{j^{*}}=\gamma_{j}^{-1}$. Henceforth we fix these matrices $\left\{\gamma_{j}\right\}_{j \in J}$ to be our preferred set of generators for $\Gamma$.

We have $\gamma_{j}\left(P_{j}\right)=P_{j^{*}+1}$ and the map $T: J \rightarrow J$ sending $j \mapsto j^{*}+1$ breaks $J$ up into finitely many orbits $[j]=\left\{j=T^{t} j, T j, T^{2} j, \ldots, T^{t-1} j\right\}$ in such a way that two vertices $P_{j}$ and $P_{j^{\prime}}$ are identified in $\mathcal{F}$ iff $j$ and $j^{\prime}$ are in the same orbit. We pick a base point $j_{0}$ in each orbit and define a partial order on $J$ by $j \prec j^{\prime}$ if $j$ and $j^{\prime}$ belong to the same orbit and $j=T^{a} j_{0}, j^{\prime}=T^{b} j_{0}$ with $0 \leq a<b<t=$ the size of the orbit.

The following construction allows us to pass from holomorphic cuspforms of weight 2 for $\Gamma$ (denoted $S_{2}(\Gamma)$ ) to homomorphisms from $\Gamma$ to the additive complex numbers $\mathbb{C}$. Fix $f \in S_{2}(\Gamma), \tau_{0} \in \mathcal{H}^{*}$ and put

$$
\Phi_{f}(\tau)=2 \pi i \int_{\tau_{0}}^{\tau} f(\xi) d \xi .
$$

Since $f$ is holomorphic $\Phi_{f}(\tau)$ is well defined and for $\gamma \in \Gamma$, the invariance of $f(\tau) d \tau$ gives

$$
\Phi_{f}(\gamma(\tau))-\Phi_{f}(\tau)=2 \pi i \int_{\tau_{0}}^{\gamma\left(\tau_{0}\right)} f(\xi) d \xi=C_{f}(\gamma) .
$$

It is easy to show (see [4]) that $C_{f}(\gamma)$ is independent of base-point $\tau_{0}$ and it defines a homomorphism $C_{f}: \Gamma \rightarrow \mathbb{C}$. Its kernel contains all elliptic and parabolic elements of $\Gamma$ as well as commutators.

3. The Petersson inner product. For $f$ and $g \in S_{2}(\Gamma)$ their Petersson inner product $\langle f, g\rangle$ is defined to be

$$
\langle f, g\rangle=\frac{i}{2} \iint_{\mathcal{F}} f(\tau) \overline{g(\tau)} d \tau \wedge \overline{d \tau} .
$$

In [9], Zagier computes the Petersson norm of $f$ in terms of the values of $C_{f}\left(\gamma_{j}\right)$ for the preferred generators $\left\{\gamma_{j}\right\}_{j \in J}$ of (see $\left.\S 2\right) \Gamma$. We show that his method works for arbitrary $f$ and $g \in S_{2}(\Gamma)$ and in so doing obtain 
THEOREM 1. With the above notation, the Petersson inner product of $f$ and $g$ in $S_{2}(\Gamma)$ is

$$
\langle f, g\rangle=\frac{i}{16 \pi^{2}}\left(\sum_{j \in J} C_{f}\left(\gamma_{j}\right) \overline{C_{g}\left(\gamma_{j}\right)}+2 \sum_{\substack{j, j^{\prime} \in J \\ j \prec j^{\prime}}} C_{f}\left(\gamma_{j}\right) \overline{C_{g}\left(\gamma_{j^{\prime}}\right)}\right) .
$$

REMARK. Let $f=g$ in (1) and keeping in mind that $\|f\|^{2}$ is real we recover the formula in [9], i.e.

$$
\|f\|^{2}=\frac{1}{8 \pi^{2}} \sum_{\substack{j, j^{\prime} \in J \\ j \prec j^{\prime}}} \operatorname{Im}\left(C_{f}\left(\gamma_{j}\right) \overline{C_{f}\left(\gamma_{j}^{\prime}\right)}\right) .
$$

On the other hand, assuming that we have $\varphi: X \rightarrow E=\mathbb{C} / \Lambda$ (see $\S 1$ ) for some lattice $\Lambda \subset \mathbb{C}$ then the holomorphic differential $d z$ on $\mathbb{C}$ being $\Lambda$ invariant defines a form on $E$. The pull-back $\varphi^{*}(d z)$ is of the form $2 \pi i f(\tau) d \tau$ for some $f \in S_{2}(\Gamma)$ and we have

$$
\begin{aligned}
&\|f\|^{2}= \frac{i}{2} \int_{\Gamma_{0}(N) \backslash \mathcal{H}} f(\tau) d \tau \wedge \overline{f(\tau)} \overline{d \tau} \\
&= \frac{i}{8 \pi^{2}} \int_{\Gamma_{0}(N) \backslash \mathcal{H}} \varphi^{*}(d z) \wedge \overline{\varphi^{*}(d z)}=\frac{i}{8 \pi^{2}} \operatorname{deg}(\varphi) \int_{E} d z \wedge \overline{d z}, \\
&\|f\|^{2}=\frac{\operatorname{deg}(\varphi)}{4 \pi^{2}} \operatorname{Vol}(E),
\end{aligned}
$$

where $\operatorname{Vol}(E)$ is the area of the fundamental period parallelogram for the lattice $\Lambda$.

Equations (2) and (3) are combined in [9] to give the following formula:

$$
\operatorname{deg}(\varphi)=\frac{1}{2 \operatorname{Vol}(E)} \sum_{\substack{j, j^{\prime} \in J \\ j \prec j^{\prime}}} \operatorname{Im}\left(C_{f}\left(\gamma_{j}\right) \overline{C_{f}\left(\gamma_{j}^{\prime}\right)}\right) .
$$

4. The action of the Hecke operators on $C_{f}$ and explicit formulas for the degree of the modular parameterization and Hecke eigenvalues of $f$. Fix $f \in S_{2}\left(\Gamma_{0}(N)\right)$ with Fourier expansion $f(\tau)=$ $\sum_{n \geq 1} a_{n} e^{2 \pi i n \tau}$, and for each prime $p$ with $(p, N)=1$, let $T(p)$ be the weight 2 Hecke operator. The operator $T(p)$ acts on the cusp-form $f$ as follows:

$$
T(p)(f(\tau))=\frac{1}{p} \sum_{l=0}^{p-1} f\left(\frac{\tau+l}{p}\right)+p f(p \tau) .
$$

Further, let the matrices $\left\{\gamma_{j}\right\}_{j \in J}$ be the preferred generators of the Hecke congruence subgroup $\Gamma_{0}(N)$. Then, for a fixed prime $p$ not dividing $N$, we have 
THEOREM 2.

$$
C_{T(p) f}\left(\gamma_{j}\right)=\sum_{k \in J} n_{p, k, j} C_{f}\left(\gamma_{k}\right) .
$$

Here $n_{p, k, j}=\sum_{l=0}^{p-1}\left(m_{k, l}+m_{k, j, l}^{\prime}\right)+m_{k, j}^{\prime \prime}$ and the integers $m_{k, l}, m_{k, j, l}^{\prime}$ and $m_{k, j}^{\prime \prime}$ are defined as follows. For a fixed $j \in J$, let $A_{l}, B_{j, l}$ and $D_{j}$ denote the matrices in $\Gamma_{0}(N)$ that map 0 to $l / p,\left(\gamma_{j}(0)+l\right) / p$ and $p \gamma_{j}(0)$ respectively. Write these matrices as a product of the standard generators, i.e.

$$
A_{l}=\prod_{\substack{i=1 \\ k \in J)}} \gamma_{k_{i}}^{\alpha_{k_{i}, l}}, \quad B_{j, l}=\prod_{\substack{i=1 \\ k \in J)}} \gamma_{k_{i}}^{\beta_{k_{i}, j, l}} \text { and } D_{j}=\prod_{\substack{i=1 \\ k \in J)}} \gamma_{k_{i}}^{\delta_{k_{i}, j}}
$$

and set

$$
m_{k, l}=\sum_{k_{i}=k} \alpha_{k_{i}, l}, \quad m_{k, j, l}^{\prime}=\sum_{k_{i}=k} \beta_{k_{i}, j, l} \quad \text { and } \quad m_{k, j}^{\prime \prime}=\sum_{k_{i}=k} \delta_{k_{i}, j} .
$$

Remark. Note that the coefficients $n_{p, k, j}$ do not depend on the cuspform $f$.

Combining Theorem 2 and equation (2), with the added assumption that the function $f$ is a newform of level $N$, results in

$$
\begin{gathered}
\langle T(p) f, f\rangle=\frac{1}{8 \pi^{2}} \sum_{\substack{j, j^{\prime} \in J \\
j \prec j^{\prime}}} \operatorname{Im}\left(C_{T(p) f}\left(\gamma_{j}\right) \overline{C_{f}\left(\gamma_{j}^{\prime}\right)}\right), \\
a_{p}\|f\|^{2}=\frac{1}{8 \pi^{2}} \sum_{\substack{j, j^{\prime} \in J \\
j \prec j^{\prime}}} \sum_{\substack{k \in J \\
j<,}} n_{p, k, j} \operatorname{Im}\left(C_{f}\left(\gamma_{k}\right) \overline{C_{f}\left(\gamma_{j}^{\prime}\right)}\right) .
\end{gathered}
$$

Now equation (3) coupled with (4) results in the following two corollaries.

COROllary 1. The pth Hecke eigenvalue (Fourier coefficient) of the cusp-form $f$ is

$$
a_{p}=\frac{1}{2 \operatorname{deg}(\varphi) \operatorname{Vol}(E)} \sum_{\substack{j, j^{\prime} \in J \\ j \prec j^{\prime}}} \sum_{k \in J} n_{p, k, j} \operatorname{Im}\left(C_{f}\left(\gamma_{k}\right) \overline{C_{f}\left(\gamma_{j^{\prime}}\right)}\right) .
$$

COROllary 2. For each prime $p$ we have

$$
\operatorname{deg}(\varphi)=\frac{1}{2 a_{p} \operatorname{Vol}(E)} \sum_{\substack{j, j^{\prime} \in J \\ j \prec j^{\prime}}} \sum_{k \in J} n_{p, k, j} \operatorname{Im}\left(C_{f}\left(\gamma_{k}\right) \overline{C_{f}\left(\gamma_{j^{\prime}}\right)}\right) .
$$

REMARKS. In [5] Goldfeld gives an algorithm for computing the numbers $C_{f}(\gamma)$ for $f \in S_{2}\left(\Gamma_{0}(N)\right)$ and $\gamma \in \Gamma_{0}(N)$ if the first $N^{2}$ Fourier coefficients of $f$ are known. Moreover, one can compute the degree of the map $\varphi$ without knowledge of the Fourier coefficients and vice-versa. 
5. A proof of Theorem 1. Starting with the definition of the Petersson inner product we compute

$$
\begin{aligned}
\langle f, g\rangle & =\frac{i}{2} \iint_{\mathcal{F}} f(\tau) \overline{g(\tau)} d \tau \wedge \overline{d \tau} \\
& =\frac{1}{4 \pi} \iint_{\mathcal{F}} d\left[\Phi_{f}(\tau) \overline{g(\tau)} \overline{d \tau}\right] \quad\left(\Phi_{f}(\tau)=2 \pi i \int_{\tau_{0}}^{\tau} f(\xi) d \xi\right) \\
& =\frac{1}{4 \pi} \int_{\partial \mathcal{F}} \Phi_{f}(\tau) \overline{g(\tau)} \overline{d \tau} \quad \text { (by Stokes' theorem) } \\
& =\frac{1}{4 \pi} \sum_{j} \int_{e_{j}} \Phi_{f}(\tau) \overline{g(\tau)} \overline{d \tau} \\
& =\frac{1}{8 \pi} \sum_{j}\left(\int_{e_{j}}+\int_{e_{j^{*}}}\right) \Phi_{f}(\tau) \overline{g(\tau)} \overline{d \tau} .
\end{aligned}
$$

Since $e_{j^{*}}$ is the image of $e_{j}$ under $\gamma_{j}$ with orientation reversed and $g(\tau) d \tau$ is $\gamma_{j}$-invariant, we have

$$
\int_{e_{j^{*}}} \Phi_{f}(\tau) \overline{g(\tau)} \overline{d \tau}=-\int_{e_{j}} \Phi_{f}\left(\gamma_{j} \tau\right) \overline{g(\tau)} \overline{d \tau}
$$

Consequently,

$$
\begin{aligned}
\langle f, g\rangle & =\frac{1}{8 \pi} \sum_{j} \int_{e_{j}}\left[\Phi_{f}(\tau)-\Phi_{f}\left(\gamma_{j} \tau\right)\right] \overline{g(\tau)} \overline{d \tau} \\
& =-\frac{1}{8 \pi} \sum_{j} \int_{e_{j}} C_{f}\left(\gamma_{j}\right) \overline{g(\tau)} \overline{d \tau} \\
& =-\frac{i}{16 \pi^{2}} \sum_{j} C_{f}\left(\gamma_{j}\right)\left[\overline{\Phi_{g}\left(P_{j+1}\right)}-\overline{\Phi_{g}\left(P_{j}\right)}\right] .
\end{aligned}
$$

Recall that $\gamma_{j^{*}}=\gamma_{j}^{-1}$ and that $C_{f}$ is a homomorphism. Replace $j$ by $j^{*}$ in the first summation to obtain

$$
\begin{aligned}
\sum C_{f}\left(\gamma_{j}\right) \overline{\Phi_{g}\left(P_{j+1}\right)} & =\sum C_{f}\left(\gamma_{j^{*}}\right) \overline{\Phi_{g}\left(P_{j^{*}+1}\right)}=\sum C_{f}\left(\gamma_{j}^{-1}\right) \overline{\Phi_{g}\left(P_{j^{*}+1}\right)} \\
& =-\sum C_{f}\left(\gamma_{j}\right) \overline{\Phi_{g}\left(\gamma_{j}\left(P_{j}\right)\right)} \\
& \left.=-\sum C_{f}\left(\gamma_{j}\right) \overline{C_{g}\left(\gamma_{j}\right)}+\overline{\Phi_{g}\left(P_{j}\right)}\right] .
\end{aligned}
$$

Inserting this in the last line of the previous computation and simplifying gives

$$
\langle f, g\rangle=\frac{i}{16 \pi^{2}} \sum_{j}\left(C_{f}\left(\gamma_{j}\right) \overline{C_{g}\left(\gamma_{j}\right)}+2 C_{f}\left(\gamma_{j}\right) \overline{\Phi_{g}\left(P_{j}\right)}\right) .
$$


Finally, we break up the second sum into orbits under $T$ (see $\S 2$ ). Let $\left[j_{0}\right]=\left\{j_{0}, T j_{0}, \ldots, T^{t-1} j_{0}\right\}$ be a typical orbit with $T^{t} j_{0}=j_{0}$. Now observe that $\sum_{j \in\left[j_{0}\right]} C_{f}\left(\gamma_{j}\right)=0$ because $\prod_{j \in\left[j_{0}\right]} \gamma_{j}$ fixes $P_{j_{0}}$ hence is the identity or an element of finite order. This observation proves fruitful as the next computation will show:

$$
\begin{aligned}
\sum_{j \in\left[j_{0}\right]} C_{f}\left(\gamma_{j}\right) \overline{\Phi_{g}\left(P_{j}\right)} & =\sum_{j \in\left[j_{0}\right]} C_{f}\left(\gamma_{j}\right)\left[\overline{\Phi_{g}\left(P_{j}\right)}-\overline{\Phi_{g}\left(P_{j_{0}}\right)}\right] \\
& =\sum_{j \in\left[j_{0}\right]} C_{f}\left(\gamma_{j}\right) \sum_{j^{\prime} \prec j} \overline{C_{g}\left(\gamma_{j^{\prime}}\right)}
\end{aligned}
$$

since $P_{j}=\left(\prod_{j^{\prime} \prec j} \gamma_{j^{\prime}}\right) P_{j_{0}}$. Combining all of this gives Theorem 1 .

6. A proof of Theorem 2. Once again we start with the definition of the action of the Hecke operator $T(p)$ on the cusp-form $f$ and compute

$$
\begin{aligned}
C_{T(p) f}\left(\gamma_{j}\right) & =2 \pi i \int_{\tau_{0}}^{\gamma_{j}\left(\tau_{0}\right)} T(p) f(\xi) d \xi \\
& =2 \pi i(\sum_{l=0}^{p-1} \int_{\tau_{0}}^{\gamma_{j}\left(\tau_{0}\right)} \underbrace{f\left(\frac{\xi+l}{p}\right) \frac{d \xi}{p}}_{\xi \mapsto p \xi^{\prime}-l}+\int_{\tau_{0}}^{\gamma_{j}\left(\tau_{0}\right)} \underbrace{f(p \xi) p d \xi}_{\xi \mapsto \xi^{\prime} / p}) \\
& =2 \pi i\left(\sum_{l=0}^{p-1} \int_{\left(\tau_{0}+l\right) / p}^{\left(\gamma_{j}\left(\tau_{0}\right)+l\right) / p} f(\xi) d \xi+\int_{p \tau_{0}}^{p \gamma_{j}\left(\tau_{0}\right)} f(\xi) d \xi\right) .
\end{aligned}
$$

We know that $C_{f}$ is independent of base-point so pick $\tau_{0}=0$ to obtain

$$
C_{T(p) f}\left(\gamma_{j}\right)=2 \pi i\left(\sum_{l=0}^{p-1}\left(\int_{l / p}^{0}+\int_{0}^{\left(\gamma_{j}(0)+l\right) / p}\right) f(\xi) d \xi+\int_{0}^{p \gamma_{j}(0)} f(\xi) d \xi\right) .
$$

Since $(p, N)=1$ it is easy to show that the rational numbers $l / p,\left(\gamma_{j}(0)+l\right) / p$ and $p \gamma_{j}(0)$ are all $\Gamma_{0}(N)$ equivalent to 0 . Hence there exist matrices $A_{l}, B_{j, l}$ and $D_{j}\left(\right.$ all in $\left.\Gamma_{0}(N)\right)$ with $A_{l}(0)=l / p, B_{j, l}(0)=\left(\gamma_{j}(0)+l\right) / p$ and $D_{j}(0)=$ $p \gamma_{j}(0)$. This gives

$$
\begin{aligned}
C_{T(p) f}\left(\gamma_{j}\right) & =2 \pi i\left(\sum_{l=0}^{p-1}\left(\int_{A_{l}(0)}^{0}+\int_{0}^{B_{j, l}(0)}\right) f(\xi) d \xi+\int_{0}^{D_{j}(0)} f(\xi) d \xi\right) \\
& =\left(\sum_{l=0}^{p-1}\left[-C_{f}\left(A_{l}\right)+C_{f}\left(B_{j, l}\right)\right]+C_{f}\left(D_{j}\right)\right) .
\end{aligned}
$$


Like any other element of $\Gamma_{0}(N)$, each of these matrices can be written in terms of the preferred generators, i.e.

$$
A_{l}=\prod_{\substack{i=1 \\ k \in J)}} \gamma_{k_{i}}^{\alpha_{k_{i}, l}}, \quad B_{j, l}=\prod_{\substack{i=1 \\(k \in J)}} \gamma_{k_{i}}^{\beta_{k_{i}, j, l}} \text { and } \quad D_{j}=\prod_{\substack{i=1 \\(k \in J)}} \gamma_{k_{i}}^{\delta_{k_{i}, j}}
$$

Since $C_{f}$ is a homomorphism this allows us to express each term in the expression (4) as a $\mathbb{Z}$-linear combination of $\left\{C_{f}\left(\gamma_{k}\right)\right\}_{k \in J}$. More precisely, for a fixed $j$ and $l$ we have

$$
\begin{gathered}
C_{f}\left(A_{l}\right)=C_{f}\left(\prod_{\substack{i=1 \\
(k \in J)}} \gamma_{k_{i}}^{\alpha_{k_{i}, l}}\right)=\sum_{k \in J} m_{k, l} C_{f}\left(\gamma_{k}\right), \\
C_{f}\left(B_{j, l}\right)=C_{f}\left(\prod_{\substack{i=1 \\
(k \in J)}}^{\beta_{k_{i}} \beta_{k_{i}, j, l}}\right)=\sum_{k \in J} m_{k, j, l}^{\prime} C_{f}\left(\gamma_{k}\right), \\
C_{f}\left(D_{j}\right)=C_{f}\left(\prod_{\substack{i=1 \\
(k \in J)}} \gamma_{k_{i}}^{\delta_{k_{i}, j}}\right)=\sum_{k \in J} m_{k, j}^{\prime \prime} C_{f}\left(\gamma_{k}\right) .
\end{gathered}
$$

Here the coefficients of $C_{f}\left(\gamma_{k}\right)$ on the right of any of these equations is the sum of all numbers that occur as an exponent of $\gamma_{k}$ in the factorization of the corresponding matrix on the left (i.e., $m_{k, l}=\sum_{k_{i}=k} \alpha_{k_{i}, l}$ etc.). The summation on the right can be taken over hyperbolic generators only since $C_{f}(\gamma)$ is zero for parabolic and elliptic elements. Combining all of this gives

$$
C_{T(p) f}\left(\gamma_{j}\right)=\sum_{k \in J}\left(\sum_{l=0}^{p-1}\left(m_{k, l}+m_{k, j, l}^{\prime}\right)+m_{k, j}^{\prime \prime}\right) C_{f}\left(\gamma_{k}\right) .
$$

Setting $n_{p, k, j}=\sum_{l=0}^{p-1}\left(m_{k, l}+m_{k, j, l}^{\prime}\right)+m_{k, j}^{\prime \prime}$ establishes Theorem 2 .

\section{References}

[1] B. Conrad, F. Diamond and R. Taylor, Modularity of certain potentially BarsottiTate Galois representations, J. Amer. Math. Soc. 12 (1999), 521-567.

[2] F. Diamond, On deformation rings and Hecke rings, Ann. of Math. (2) 144 (1996), 81-100.

[3] G. Frey, Links between elliptic curves and certain diophantine equations, J. Indian Math. Soc. 51 (1987), 117-145.

[4] D. Goldfeld, Modular elliptic curves and Diophantine problems, in: Number Theory (Banff, AB, 1988), de Gruyter, New York, 1990, 157-175.

[5] - On the computational complexity of modular symbols, Math. Comp. 58 (1992), 807-814.

[6] R. Taylor, Icosahedral Galois representations, in: Olga Taussky-Todd: In memoriam, Pacific J. Math., Special Issue, 1997, 337-347. 
[7] R. Taylor and A. Wiles, Ring-theoretic properties of certain Hecke algebras, Ann. of Math. (2) 141 (1995), 553-572.

[8] A. Wiles, Modular elliptic curves and Fermat's last theorem, ibid., 443-551.

[9] D. Zagier, Modular parameterization of elliptic curves, Canad. Math. Bull. 28 (1985), 372-384.

Fixed Income Research

Credit Suisse First Boston

11 Madison Avenue

New York, NY, 10010, U.S.A.

E-mail: arjune.budhram@csfb.com 